\title{
A Methodology to Forecast Commodity Prices in Vietnam
}

\author{
Thi Ngoc Trang Nguyen ${ }^{1} \&$ Ngoc Tho Tran ${ }^{1}$ \\ ${ }^{1}$ School of Finance, University of Economics Ho Chi Minh City, Vietnam \\ Correspondence: Thi Ngoc Trang Nguyen, School of Finance, University of Economics Ho Chi Minh City, Ho \\ Chi Minh City, Vietnam. E-mail: trangtcdn@ueh.edu.vn \\ Received: February 22, 2015 \\ Accepted: March 7, 2015 \\ Online Published: April 25, 2015 \\ doi:10.5539/ijef.v7n5p44 \\ URL: http://dx.doi.org/10.5539/ijef.v7n5p44
}

\begin{abstract}
Risk management of commodity prices is an important yet challenging task. Given the complex behaviour of commodity prices, this creates the need of using sophisticated models of commodity prices dynamics. Obviously, parameter estimation of such models poses another challenge. Previous literature has addressed this problem using Markov Chain Monte Carlo, which is computationally expensive for parameter estimation and inference. In this paper we develop an efficient Maximum Likelihood Estimation procedure based on the characteristic function. We then estimate parameters a stochastic volatility model with stochastic drift utilizing the time-series of rice and coffee prices. We show that such model produces realistic distributions of both commodity prices. Finally, using the estimated model parameters we calculate various risk measures such as Value at Risk or Expected Shortfall.
\end{abstract}

Keywords: risk management, commodities, maximum likelihood

\section{Introduction}

Commodities are broadly defined as (physical) products which can be traded on the market. Examples of commodities include energy, metals or agricultural products such as coffee and rice. These products are fundamentally important to the world economy, which is driven by increasing demand as well as political factors including market regulation.

Buyers and sellers can trade commodities in the spot market as well as the derivatives market. Market participants who understand the behaviour of commodity prices are more likely to have more realistic expectations about future price movements. Solid understanding of commodity markets is also important for companies seeking to hedge their underlying price risk as well as those investing in commodities. Commodity investments differ from stocks and bonds in that they cannot produce a stream of cash flows. Historically, commodity prices increase with inflation, which makes commodities a good hedge against inflation. Commodities offer diversification benefits because commodity returns tend to have low correlation with other asset classes. Over the past decade, commodities have become increasingly popular with many institutional investors, as part of their diversification strategies.

Many commodity markets are characterised by spikes, seasonality, mean-reversion and non-constant volatility. The non-constant volatility observed in commodity markets is a motivation for stochastic volatility model of commodity prices. Commodity prices exhibit significant time-varying volatility because elasticities of short term production and consumption are low. Volatility of commodity prices can reduce the profit of business or investment strategies and negatively affect consumers. Observed variability of commodity prices elevates importance of risk management techniques to monitor, measure and manage commodity risk. Effective risk management requires thorough understanding of commodity price behaviour and sophisticated modelling of commodity prices. Demand for robust and effective ways of risk management is also stimulated by the growth of commodity market.

Managing commodity risk poses a challenge for both producers and consumers of commodities. Because of the nature of their business, produces hold commodities in stock. Consequently, their operating cash-flow is affected by commodity price changes. As for consumers, they are in general price-takers and therefore their net cash-flow is also affected by fluctuations of commodity prices.

The remainder of this paper is organized as follows. Section 2 reviews related literature. Section 3 describes the data and methodology. Results are presented in Section 4. Finally, Section 5 concludes the paper. 


\section{Literature Review}

It is a well know fact that volatility of the financial time-series is time-varying, which has been already shown by Engle (1982). Moreover, financial time-series exhibits what is known as volatility clustering, meaning that large (small) moves are followed by large (small) moves. Another two important stylized facts of the financial time-series are heavy tails and non-zero skewness of the returns distribution.

Stochastic volatility models have been developed to capture stylized facts of the financial time-series with most notable contributions from Wiggins (1987), Hull and White (1987, 1988), Scott (1987), Stein and Stein (1991) and Heston (1993). The most pronounced difference between these models lies in the specification of the volatility process. Scott (1987), Wiggins (1987) and Stein and Stein (1991) all assume that the logarithm of the variance follows a mean-reverting Ornstein-Uhlenbeck process. In the Hull and White model, the variance process follows a geometric Brownian motion with the volatility of volatility being a linear function of the instantaneous variance. On the other hand, the variance process in the Heston model is given by a mean-reverting Cox-Ingersoll-Ross process with the volatility of volatility being a linear function of the instantaneous volatility.

The idea to use state of art, yet mathematically tractable stochastic volatility models to model commodity prices is not new. In fact, Brooks and Prokopczuk (2013) apply the Heston, Bates, as well as Duffie, Pan, and Singleton (2000) models to commodity prices. The Bates (1996) model extends the Heston model to accommodate jumps following a compound Poisson process in the stock price process. The SVCJ model of Duffie, Pan, and Singleton (2000) assumes that both the price and volatility jump simultaneously, which is motivated by the fact that price jumps are often accompanied volatility jumps. Liu, Chng and $\mathrm{Xu}$ (2014) analyse hedge ratios of commodity prices generated by these three models. Benth (2011) applies the Heston model as well as the Barndorff-Nielsen and Shephard model to commodity prices. Brooks and Prokopczuk (2013) as well as Liu, Chng and Xu (2014) use the Markov Chain Monte Carlo (MCMC) to estimate the parameters of these models. Solibakke (2014) uses a multifactor stochastic volatility model for the carbon prices, also applying MCMC for parameter estimation and inference. MCMC algorithms are in general computationally expensive to achieve a high degree of accuracy. The fact that MCMC is computationally intensive for parameter estimation and inference is also acknowledged by Solibakke (2014). As an alternative to MCMC, a Maximum Likelihood method can be used to estimate the model parameters.

In the option pricing context, Trolle and Schwartz propose a model based on the Heath, Jarrow, and Morton (1992) framework. In their model futures prices are driven by three factors while option prices are driven by two additional volatility factors. The model parameters are estimates using quasi-maximum likelihood and extended Kalman filter. Yan (2002) proposes a multivariate stochastic volatility model that considers stochastic volatility, stochastic interest rates, stochastic convenience yields as well as simultaneous jumps in the spot price and volatility. In this model, the volatility follows a square-root jump-diffusion process whereas the interest rate follows a square-root process and the convenience yield follows an Ornstein-Uhlenbeck process. Hikspoors and Jaimungal (2008) assume that stochastic volatility in a factor commodities spot price model follows mean-reverting Ornstein-Uhlenbeck process. Li and Linetsky (2014) model commodity prices using subordinate Ornstein-Uhlenbeck process. Their model also features stochastic volatility and mean-reverting jumps.

In the energy market context, Eydeland and Geman (1998) were among the first to use the Heston model. Barndorff-Nielsen, Benth and Veraart (2013) introduce volatility modulated Lévy-driven Volterra processes for modelling energy prices. Their model allows for jumps as well as stochastic volatility. They point out that the latter is a key factor in modelling of energy prices. Benth and Vos (2013) propose a multivariate stochastic volatility model for energy prices. The model is able to capture stochastic volatility, price spikes and mean-reversion of prices observed in the energy markets.

\section{Data and Methodology}

We obtain monthly time-series of rice (summer-autumn crop) and robusta coffee beans prices from February 2009 until November 2014 from DataStream. The time period considered is limited by the data avaialbility. Both time-series are denominated in Vietnam Dong (VND). We choose rice and coffee as these are by far the two most important commodities produced in Vietnam. Summary statistics of the data are presented in Table 1. 
Table 1. Summary statistics

\begin{tabular}{lllllll}
\hline & Mean & Variance & Skewness & Kurtosis & Minimum & Maximum \\
\hline Rice & 5562 & 867842 & -0.7652 & 3.2215 & 3050 & 7550 \\
Coffee & 35732 & 65339640 & -0.2320 & 1.9524 & 22350 & 50700 \\
\hline
\end{tabular}

We conclude that both price distributions are skewed to the left. The distribution of rice price is leptokurtic, meaning it is peaked and has fat tails, whereas the distribution of coffee price is platykurtic, meaning it is less peaked and has thinner tails. This empirical evidence motivates the validity of a stochastic volatility model is to capture the distribution of both rice and coffee prices.

Heston model with stochastic interest rates has been introduced in Grzelak and Oosterlee (2011). The dynamics of the model is given by the following set of stochastic differential equations:

$$
\begin{gathered}
d S_{t}=r_{t} S_{t} d t+\sqrt{V_{t}} S_{t} d W_{t}^{S} \\
d v_{t}=\kappa\left(\mu-v_{t}\right) d t+\gamma \sqrt{V_{t}} d W_{t}^{v} \\
d r_{t}=\lambda\left(\theta-r_{t}\right) d+\eta r_{t}^{p} d W_{t}^{r}
\end{gathered}
$$

where $<\mathrm{dW}_{\mathrm{t}}^{\mathrm{S}} \mathrm{dW}_{\mathrm{t}}^{\mathrm{v}}>=\rho_{\mathrm{S}, \mathrm{v}} \mathrm{dt}, \quad<\mathrm{dW}_{\mathrm{t}}^{\mathrm{S}} \mathrm{dW}_{\mathrm{t}}^{\mathrm{r}}>=\rho_{\mathrm{S}, \mathrm{r}} \mathrm{dt}$ and $\left\langle\mathrm{dW}_{\mathrm{t}}^{\mathrm{S}} \mathrm{dW}_{\mathrm{t}}^{\mathrm{v}}>=0\right.$. In the above equation, $\mathrm{S}_{\mathrm{t}}$ denotes the commodity price, $r_{t}$ denotes the drift, $v_{t}$ denotes the variance, $\kappa$ denotes the variance mean-reversion, $\mu$ denotes the long-term variance, $\gamma$ denotes the volatility of volatility, $\lambda$ denotes the drift mean-reversion, $\theta$ denotes the long-term drift and $\eta$ denotes the volatility of drift. $\rho_{\mathrm{S}, \mathrm{v}}$ is the correlation between commodity price changes and changes in variance while $\rho_{\mathrm{S}, \mathrm{r}}$ is the correlation between commodity price changes and changes in drift. For the Heston-Hull-White model $\mathrm{p}=0$ whereas for the Heston-Cox-Ingersoll-Ross model $\mathrm{p}=0.5$. It is assumed that the correlation between changes in the variance and changes in drift is 0 .

It is important to note that the parameters of the variance process in the Heston-Cox-Ingersoll-Ross model have a meaningful economic interpretation. For example, the mean-reversion parameter determines the degree of volatility clustering. Correlation between commodity price and variance processes affects the heaviness of the tails. On the other hand, the peakness of the distribution depends on the volatility of volatility.

As shown in Grzelak and Oosterlee (2011), for the above model it is possible to approximate the characteristic function by:

$$
\psi(u)=\exp \left(A(u, \tau)+B(u, \tau) x_{0}+C(u, \tau) r_{0}+D(u, \tau) v_{0}\right)
$$

where $\mathrm{x}_{0}=\log \left(\mathrm{S}_{0}\right)$ and $\tau=\mathrm{T}$-t. We refer to Grzelak and Oosterlee (2011) for details.

The probability density function (pdf) can be obtained as follows. First, let us use the following inversion formula:

$$
\operatorname{Pr}\left(S_{t}>s\right)=\frac{1}{2}+\frac{1}{\pi} \int_{0}^{\infty} \operatorname{Re}\left[\frac{\exp (-i \omega \log (s)) \psi(\omega)}{i \omega}\right] d \omega
$$

where $\psi(\omega)$ is the characteristic function. By definition, the cumulative distribution function (cdf) is given by:

$$
F(s)=\operatorname{Pr}\left(S_{t} \leq s\right)=1-\operatorname{Pr}\left(S_{t}>s\right)
$$

Finally, differentiating $\mathrm{F}(\mathrm{s})$ respect to s yields the pdf:

$$
f(s)=\frac{1}{2}+\frac{1}{s \pi} \int_{0}^{\infty} \operatorname{Re}[\exp (-i \omega \log (s)) \psi(\omega)] d \omega
$$

which can be evaluated numerically using the Gaussian quadrature. Having the pdf, it is also possible to use Maximum Likelihood Estimator to obtain the model parameters.

We note that the Heston model with stochastic interest rates is an affine model. This is because its characteristic function is an exponential of an affine function of the state variables. The class of affine models is very rich and contains many financial models such as Black-Scholes, Merton, Variance Gamma, Heston, Bates or Carr Geman Madan Yor to name but a few. In fact, the methodology presented above applies not only to the Heston model with stochastic interest rates, but extends to the whole class of affine models. 
The Maximum Likelihood Estimation procedure outlined above has extensive applications in risk management. For example, resulting model parameters can be used to project the distribution of risk factors over a given time horizon. Moreover, using numerical inversion of the cdf, it is possible to calculate risk measures such as Value at Risk (VaR) or Expected Shortfall (ES).

\section{Results}

We apply the methodology described in the previous section to estimate the parameters of the Heston model with stochastic interest rates. We note that it takes about 10 seconds to estimate the model parameters on a laptop with the $17-3610 \mathrm{QM} 2.3 \mathrm{GHz}$ processor and 16Gb RAM. Between the Heston-Hull-White model and the Heston-Cox-Ingersoll-Ross model the latter is preferred as it has lower Akaike information criterion. The estimated model parameters are presented in Table 2.

Table 2. Estimated model parameters

\begin{tabular}{lllllllllll}
\hline & $\mathrm{r}_{0}$ & $\mathrm{v}_{0}$ & $\kappa$ & $\mu$ & $\gamma$ & $\lambda$ & $\theta$ & $\eta$ & $\rho_{\mathrm{S}, \mathrm{v}}$ & $\rho_{\mathrm{S}, \mathrm{r}}$ \\
\hline Rice-2M & 0.063 & 0.006 & 68.38 & 0.013 & 1.879 & 17.56 & 0.103 & 0.003 & -0.885 & -0.124 \\
Rice & 0.285 & 0.016 & 69.22 & 0.037 & 1.810 & 19.68 & 0.102 & 0.004 & -0.420 & -0.053 \\
Rice+2M & 0.204 & 0.019 & 64.29 & 0.042 & 1.337 & 18.86 & 0.103 & 0.005 & -0.525 & -0.050 \\
Coffee-2M & 0.045 & 0.021 & 47.01 & 0.020 & 0.007 & 15.61 & 0.114 & 0.046 & -0.386 & -0.067 \\
Coffee & 0.048 & 0.058 & 45.46 & 0.022 & 0.102 & 19.68 & 0.088 & 0.002 & -0.476 & -0.025 \\
Coffee+2M & 0.043 & 0.009 & 47.82 & 0.019 & 0.008 & 17.47 & 0.103 & 0.195 & -0.419 & -0.122 \\
\hline
\end{tabular}

For both rice and coffee, the speed of mean-reversion of the variance process is much higher than that of the drift process. Moreover, the variance process has usually higher volatility, but lower long-term compared to the drift process. Finally, while both price processes are negatively correlated with the commodity price changes, it is the variance process that exhibits more negative correlation to changes in the commodity price. To investigate the robustness of the estimated model parameters we extended or reduced the length of the sample period by two months and then reestiamted the model. The results of the robustness checks are also presented in Table 2. For example, the first row of Table 2 reports the estimated model parameters for rice when the sample period is reduced by two months. Similarly, the last row of this table reports the estimated model parameters for coffee when the sample period is extended by two months. We note that the estimated model parameters are remarkably robust as they do not change much when extending or reducing the length of the sample period.
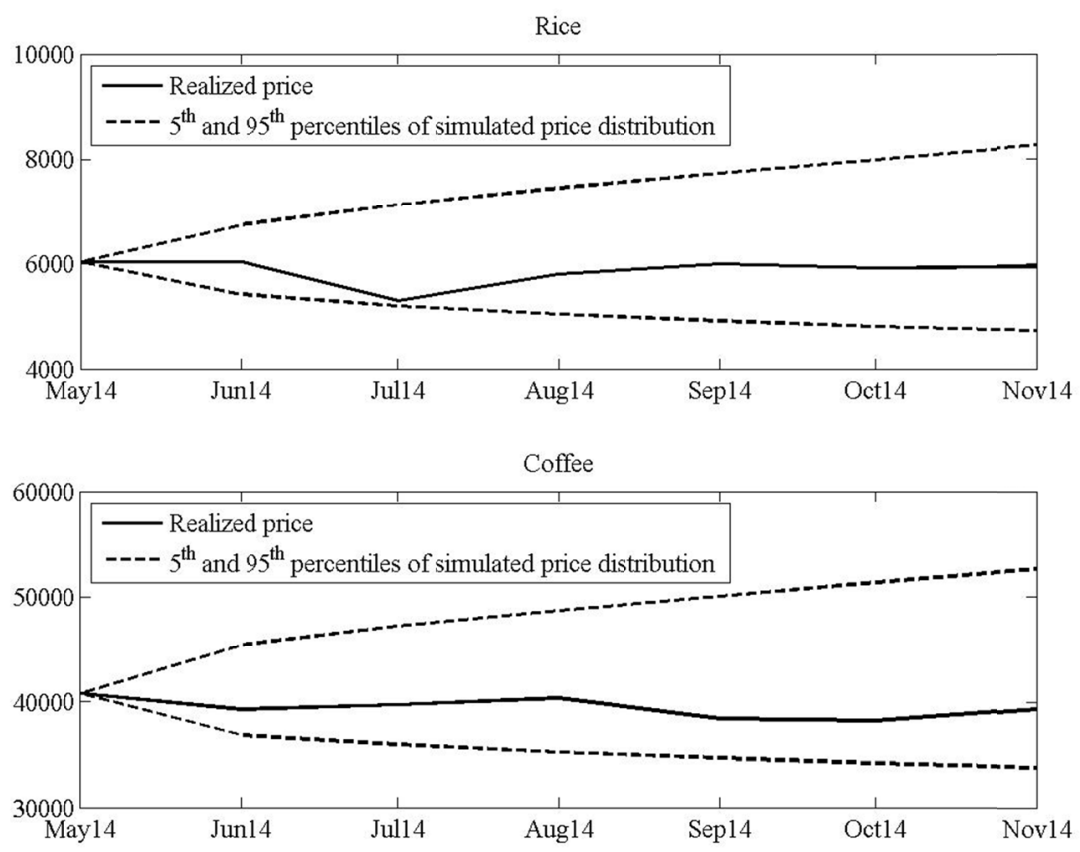

Figure 1. Realized commodity prices as well as 5th and 95th percentiles of simulated price distributions 
We note that in the above experiment we only used 64 monthly observations out of 70 , because in the next experiment we would like to perform a simulation study to backtest the model. The idea is to forecast 6 months forward coffee price at May 2014 and compare the realized coffee price with the model forecasts. These forecasts provide information about the likelihood of rising and falling price scenarios occurring. Such information is particularly useful for risk management in that the price risk can be quantified.

In Figure 1 we display the realizations as well as the 5th and 95th percentiles of simulated rice and coffee price distributions obtained using 100,000 Monte Carlo simulations.

Figure 1 clearly shows that the realized rice and coffee prices lie between the 5th and 95th percentiles of simulated price distributions. In summary, Heston-Cox-Ingersoll-Ross model produces realistic distributions of risk factors over a given time horizon and thus is suitable for risk management purposes.

Now we turn to calculation of two risk measures, namely Value at Risk (VaR) and Expected Shortfall (ES). The first one is a statistical measure of downside risk expressed in monetary terms. It is the maximum loss over a target horizon estimated with a given level of confidence. For example, with $99 \%$ confidence, there is a less than $1 \%$ chance that the actual loss will be larger than the VaR. Another key part of the VaR definition is the holding period. The choice of holding period will depend on how the resultant VaR is to be used. VaR is a key measure in risk management. A complementary risk measure to VaR is the ES. It has an advantage over VaR of being a coherent risk measure, because it is not only monotonic, invariant under translation, and homogeneous, but also sub-additive. ES is calculated as an average of losses that are larger or equal to VaR. 5\% VaR estimated at May 2014 over 6 months horizon is 1258 (rice) and 6198 (coffee). It is calculated as a difference between the coffee price at May 2014 minus 5th percentile of the inverse cdf of the Heston-Cox-Ingersoll-Ross model, with parameters given in Table 2, evaluated at the probability of 5\%. On the other hand, 5\% ES estimated at May 2014 over 6 months horizon is 1538 (rice) and 7612 (coffee). It is calculated as an average of VaR over various percentile levels. For comparison, the loss realized over 6 months on a portfolio consisting of one unit of rice is 90, whereas the loss realized over 6 months on a portfolio consisting of one unit of coffee is 1450 .

\section{Conclusion}

In this paper we address the problem of commodity risk management. Proper commodity risk management is a matter of significant importance for commodity investors as well as firms that produce and consume commodities. Complex behaviour of commodity prices requires sophisticated models of commodity prices dynamics. Parameter estimation of such models is a difficult task. Previous literature has addressed this problem using Markov Chain Monte Carlo, which requires large computational effort. This paper contributes to the existing body of literature by developing an efficient Maximum Likelihood Estimation procedure based on the characteristic function to estimate parameters of affine models. We use it to estimate parameters of the Heston-Cox-Ingersoll-Ross model utilizing the time-series of rice and coffee prices. Estimating the model we find negative link between price volatility and the commodity price changes. We also find that the speed of mean-reversion of the variance process is much higher than that of the drift process. Moreover, we demonstrate that this model produces realistic distributions of both commodity prices. Finally, we use estimated model parameters to calculate various risk measures such as Value at Risk or Expected Shortfall.

\section{Acknowledgements}

This research was funded by a grant from University of Economics Ho Chi Minh City.

\section{References}

Barndorff-Nielsen, O., Benth, F. E., \& Veraart, A. E. D. (2013). Modelling energy spot prices by volatility modulated lévy-driven volterra processes. Bernoulli, 19(3), 803-845. http://dx.doi.org/10.3150/12-BEJ476

Bates, D. (1996). Jumps and stochastic volatility: The exchange rate processes implicit in deutschemark options. Review of Financial Studies, 9(1), 69-107. http://dx.doi.org/10.1093/rfs/9.1.69

Benth, F. E. (2011). The stochastic volatility model of barndorff-nielsen and shephard in commodity markets. Mathematical Finance, 21(4), 595-625. http://dx.doi.org/10.1111/j.1467-9965.2010.00445.x

Benth, F. E., \& Vos, L. (2013). Cross-commodity spot price modelling with stochastic volatility and leverage for energy markets. Advances in Applied Probability, 45(2), 545-571. http://dx.doi.org/10.1239/aap/1370870129

Brooks, C., \& Prokopczuk, M. (2013). The dynamics of commodity prices. Quantitative Finance, 13(4), $527-542$. http://dx.doi.org/10.1080/14697688.2013.769689

Duffie, D., Pan, J., \& Singleton, K. (2000). Transform analysis and asset pricing for affine jump-diffusions. 
Econometrica, 68(6), 1343-1376. http://dx.doi.org/10.1111/1468-0262.00164

Engle, R. F. (1982). Autoregressive conditional heteroscedasticity with estimates of the variance of united kingdom inflation. Econometrica, 50(4), 987-1007. http://dx.doi.org/10.2307/1912773

Eydeland, A., \& Geman, H. (1998). Pricing power derivatives. Risk, 71-73.

Grzelak, L., \& Oosterlee, C. (2011). On the heston model with stochastic interest rates. SIAM Journal on Financial Mathematics, 2(1), 255-286. http://dx.doi.org/10.1137/090756119

Heath, D., Jarrow, R., \& Morton, A. (1992). Bond pricing and the term structure of interest rates: A new methodology for contingent claims valuation. Econometrica, 60(1), 77-105. http://dx.doi.org/10.2307/2951677

Heston, S. L. (1993). A closed solution for options with stochastic volatility, with application to bond and currency options. Review of Financial Studies, 6(2), 327-343. http://dx.doi.org/10.1093/rfs/6.2.327

Hikspoors, S., \& Jaimungal, S. (2008). Asymptotic pricing of commodity derivatives for stochastic volatility spot models. Applied Mathematical Finance, 15(5), 449-477. http://dx.doi.org/10.1080/13504860802170432

Hull, J., \& White, A. (1987). The pricing of options on assets with stochastic volatilities. Journal of Finance, 42(2), 281-300. http://dx.doi.org/10.2307/2328253

Hull, J., \& White, A. (1988). An analysis of the bias in option pricing caused by a stochastic volatility. Advances in Futures and Options Research, 3, 27-61.

Li, L., \& Linetsky, V. (2014). Time-changed ornstein-uhlenbeck processes and their applications in commodity derivative models. Mathematical Finance, 24(2), 289-330. http://dx.doi.org/10.1111/mafi.12003

Liu, Q., Chng, M. T., \& Xu, D. (2014). Hedging industrial metals with stochastic volatility models. Journal of Futures Markets, 34(8), 704-730. http://dx.doi.org/10.1002/fut.21671

Scott, L. O. (1987). Option pricing when the variance changes randomly: Theory, estimation and an application. Journal of Financial and Quantitative Analysis, 22, 419-438. http://dx.doi.org/10.2307/2330793

Solibakke, P. B. (2014). Scientific stochastic volatility models for the european carbon markets: Forecasting and extracting conditional moments. International Journal of Business, 19(1), 63-98.

Stein, E., \& Stein, C. J. (1991). Stock priced distributions with stochastic volatility: An analytical approach. Review of Financial Studies, 4(4), 727-752. http://dx.doi.org/10.1093/rfs/4.4.727

Trolle, A. B., \& Schwartz, E. S. (2009). Unspanned stochastic volatility and the pricing of commodity derivatives. Review of Financial Studies, 22(11), 4423-4461. http://dx.doi.org/10.1093/rfs/hhp036

Wiggins, J. B. (1987). Option values under stochastic volatility: Theory and empirical estimates. Journal of Financial Economics, 19, 351-372. http://dx.doi.org/10.1016/0304-405X(87)90009-2

Yan, X. (2002). Valuation of commodity derivatives in a new multi-factor model. Review of Derivatives Research, 5(3), 251-271. http://dx.doi.org/10.1023/A:1020871616158

\section{Copyrights}

Copyright for this article is retained by the author(s), with first publication rights granted to the journal.

This is an open-access article distributed under the terms and conditions of the Creative Commons Attribution license (http://creativecommons.org/licenses/by/3.0/). 